\title{
The Scattering Constant for Multiply Charged Particles in Photographic Emulsion.
}

C. Fichtel and M. W. Friediander

(Nuovo Cimento, 10, 1032 (1958))

\author{
E R R A T
}

Figs. 1 and 2 illustrate the variation of scattering constant with cell-size and velocity for protons and $\alpha$-particles.

The labelling of the curves with respect to velocity has been inverted: the curves labelled $\beta=1.0$ should be labelled $\beta=0.5$, and vice versa for the curves labelled $\beta=0.5$. 